\title{
SOP 02: Evaluation and Selection of a Clinical Trial Proposal for CESAR
}

\section{Background and Principles}

SOP 2 defines the composition and procedures of the Project Review Committee (PRC) of CESAR. The PRC works independently of the time schedule of working group meetings, and its task is to ensure a rapid and objective evaluation of trial projects proposed to CESAR via its working groups and the prompt activation of approved studies. The PRC's decision must take into consideration the interests of CESAR to have adequate information on the investigational drug and the proposed project while at the same time maintaining the strictest secrecy of confidential information provided by a sponsor.

\section{Membership of the Project Review Committee}

The membership of the PRC consists of the chairpersons and/or co-chairpersons of the working groups CESARPhase I, CESAR-Phase II, and CESAR-Phase III and of statistician. For a given project, e.g. a phase II study, the chairperson and co-chairperson of the respective working group will finally decide who will act as 'Working Group Representative of the PRC' for that project.

Eligibility Criteria for Membership in the Protocol Review Committee of CESAR

To be eligible for being elected as a member of the PRC in CESAR, the candidate must fulfill the following criteria:

- Holding an academic degree (MD, PhD);

- Proper knowledge of GCP guidelines, statistical methodology and ethical regulations (e.g. Helsinki declaration);

- Documented major involvement in the development of protocols for clinical phase I, phase II and/or phase III studies according to GCP guidelines;

- Experience as clinical investigator or co-investigator in monocenter and multicenter clinical trials and first-, senior- or co-authorship of more than three publications in peer-reviewed journals reporting results from such clinical studies;

- Disclosure of financial interests in non-academic organizations and/or private companies.

\section{Tasks of the Project Review Committee}

The PRC evaluates proposed trial projects on the basis of a standardized checklist (see Attachment 1). The PRC's decision will in particular be based on the following criteria:

- Adequate pharmaceutical, pharmacological, and toxicological characterization of the investigational product,

- The proposed design and draft of the trial protocol submitted by the sponsor (including information to be given to the patients),

- The risk/benefit assessment regarding the use of the investigational product,

- Compatibility of the trial with the global objectives of CESAR,

- Feasibility of the trial for the institutions represented in CESAR or its working groups,

- Scientific priority of the trial project (novel investigational product / adequate trial design).

Upon approval of a proposed trial, the PRC in consultation with the members of the corresponding working group will coordinate the finalization of the study documentation. The identification of the institutions to participate in the study will finally be performed by the chairperson(s) of the respective working group(s).

\section{Evaluation Process}

Offers by Sponsors

A sponsor who suggests a study project has to submit the relevant documentation in sufficient number of $\mathrm{x}$ copies to 
the Chair of the relevant working group (5 sets of copies for the PRC, one set for the Coordinating Investigator (CI) to be nominated for the project). Checklist 2a (see Attachment 1), to be completed by the sponsor, serves to facilitate and speed up the formal evaluation process of the proposed study.

\section{Evaluation by the PRC}

The Chair of the PRC will distribute the materials to the members of the PRC and will

- Set a deadline for the return of the members' comments (using the assessment section of Checklist 2a) and

- Schedule meetings of the committee.

It is at the discretion of the PRC to invite to its meetings representatives from the sponsor's institution.

\section{Preliminary Decision by the PRC}

- The PRC will evaluate a proposed study and then decide on its adoption or rejection.

- When a project has been rejected by the PRC, the PRC chairperson or co-chairperson will present and defend the decision either at the next regular meeting of the working group or within 4 weeks after the application.

- If the PRC is undecided, it may postpone its decision until the next regular meeting of the working group, at which the reasons against an immediate adoption have to be given. The members of the working group who are present at the meeting will then adopt or reject the proposal by simple majority vote.

- Upon an affirmative decision by the PRC, its Chair will immediately forward to the CI the complete data material submitted by the sponsor (including the Investigator's Brochure). The Chair should strive for a well-balanced consideration of all working group members in the nomination of CIs.

\section{Notification of the Working Group Members}

Unless the trial protocol already contained a comprehensive summary ('synopsis') that complies with the templates shown in Attachments 1 and 2 of 'SOP 03: Preparation and Structure of Trial Protocols', the CI will write such a summary and distribute it to all working group members via the PRC chairperson. Each working group member may request the full version of the trial protocol within 4 weeks (unless not otherwise agreed by contract).

\section{Consent on the Protocol among Clinical Investigators}

Working group members who wish to participate in a given study have to complete Checklist 2b (see Attach- ment 2) and return it to the PRC Chairperson within 2 weeks (unless otherwise specified). On this checklist, the investigator candidates may suggest modifications to the study protocol.

The CI will then incorporate suggested modifications, in concert with the sponsor. The sponsor and the chairperson(s) of the respective Working Group(s), will jointly determine a maximal number of centers that should reasonably participate in the study.

It is the responsibility of the CI that the final version of the study protocol complies with change requests of both PRC members and participating working group members. In case of considerable, and in particular conflicting, wishes for changes, the PRC chairperson and the chairperson of the Working Groups will jointly decide whether a second round of the above consent procedure may be necessary.

\section{Ethics Committee Approval}

The CI applies for the vote of the Ethics Committee relevant for the local institution. Upon approval by the Ethics Committee, the CI will forward a copy of the decision and the trial protocol (and any amendment) to the sponsor and the PRC chairperson.

\section{Study Activation}

The chairpersons of the respective working groups will circulate the final version of the synopsis of the trial protocol to all members of the respective working group (CESARPhase I, CESAR-Phase II, CESAR-Phase III) and thus activate the study.

The CI will present the study at the next scheduled meeting of the Working Group.

It is the obligation of the sponsor to ensure compliance with applicable local regulatory requirements before it supplies trial medication to the investigator's site and authorizes the use of the product in humans (for details see 'SOP 6: Selecting the Participating Centers and Activation of a Trial').

\section{Appendix}

Attachment 1: Checklist 2a: Project Evaluation by the Project Review Committee (PRC)

Attachment 2: Checklist 2b: Vote on Project/Trial Protocol by PRC Members

Attachment 3: Flow of Information during Protocol Review 

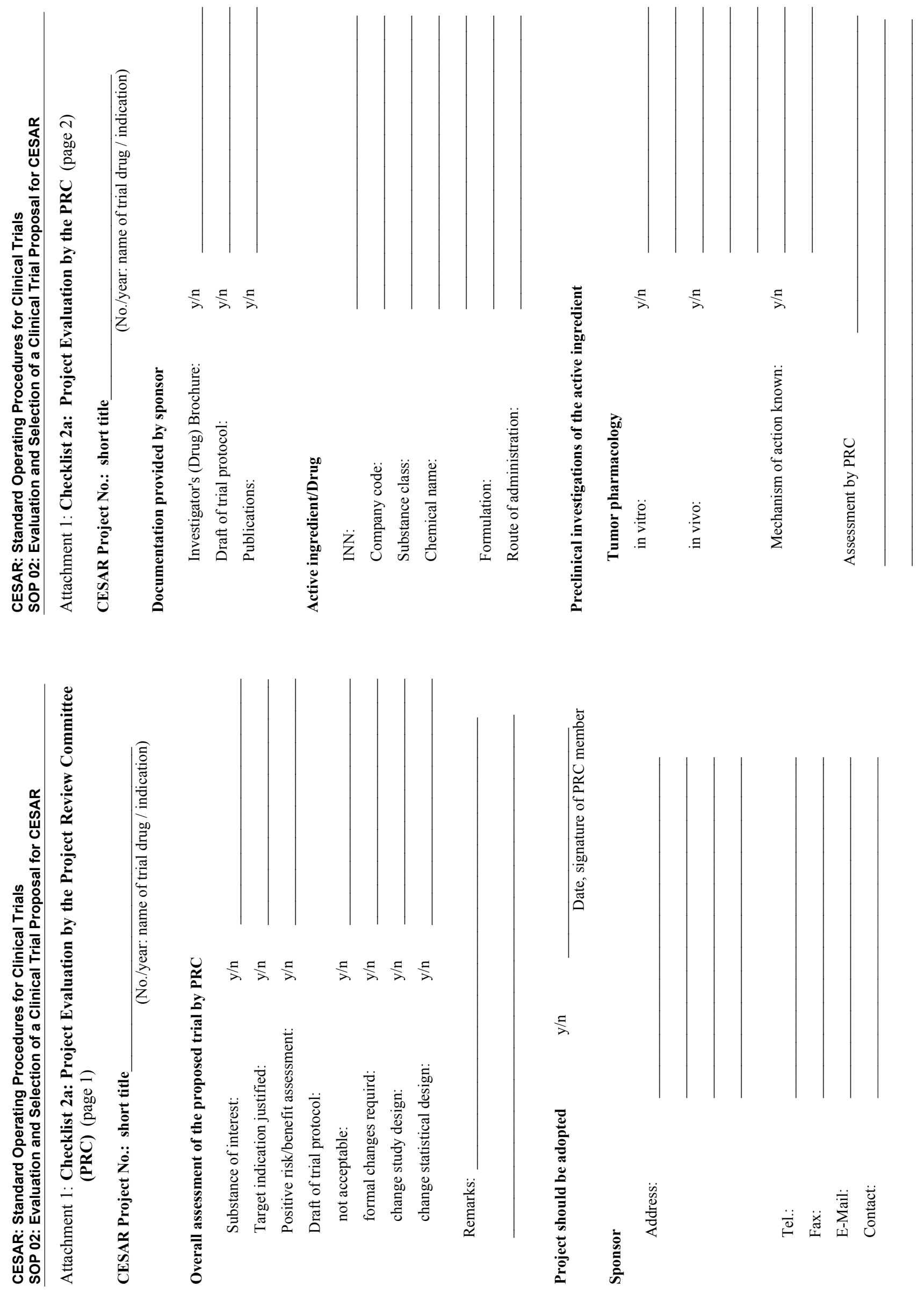

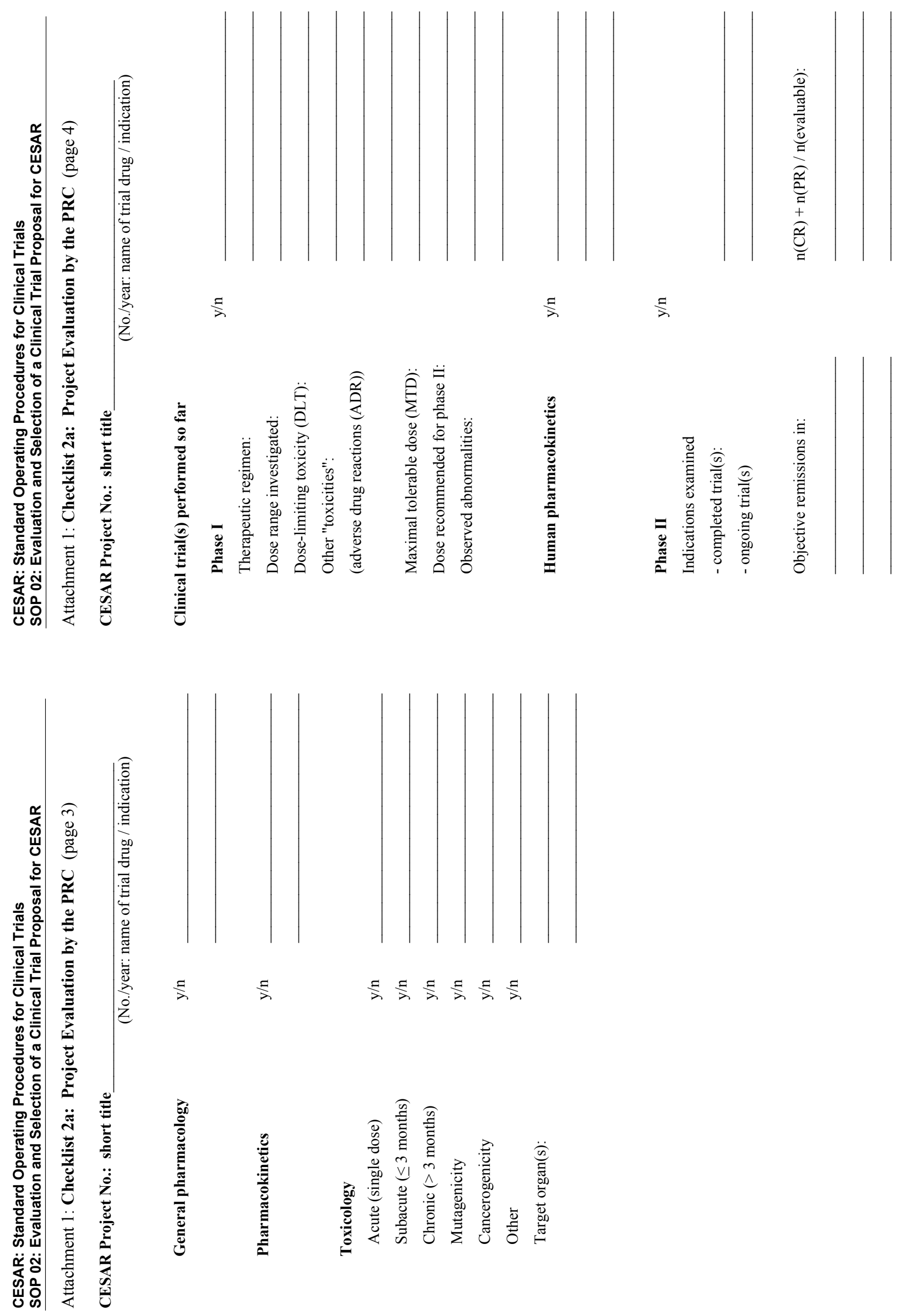

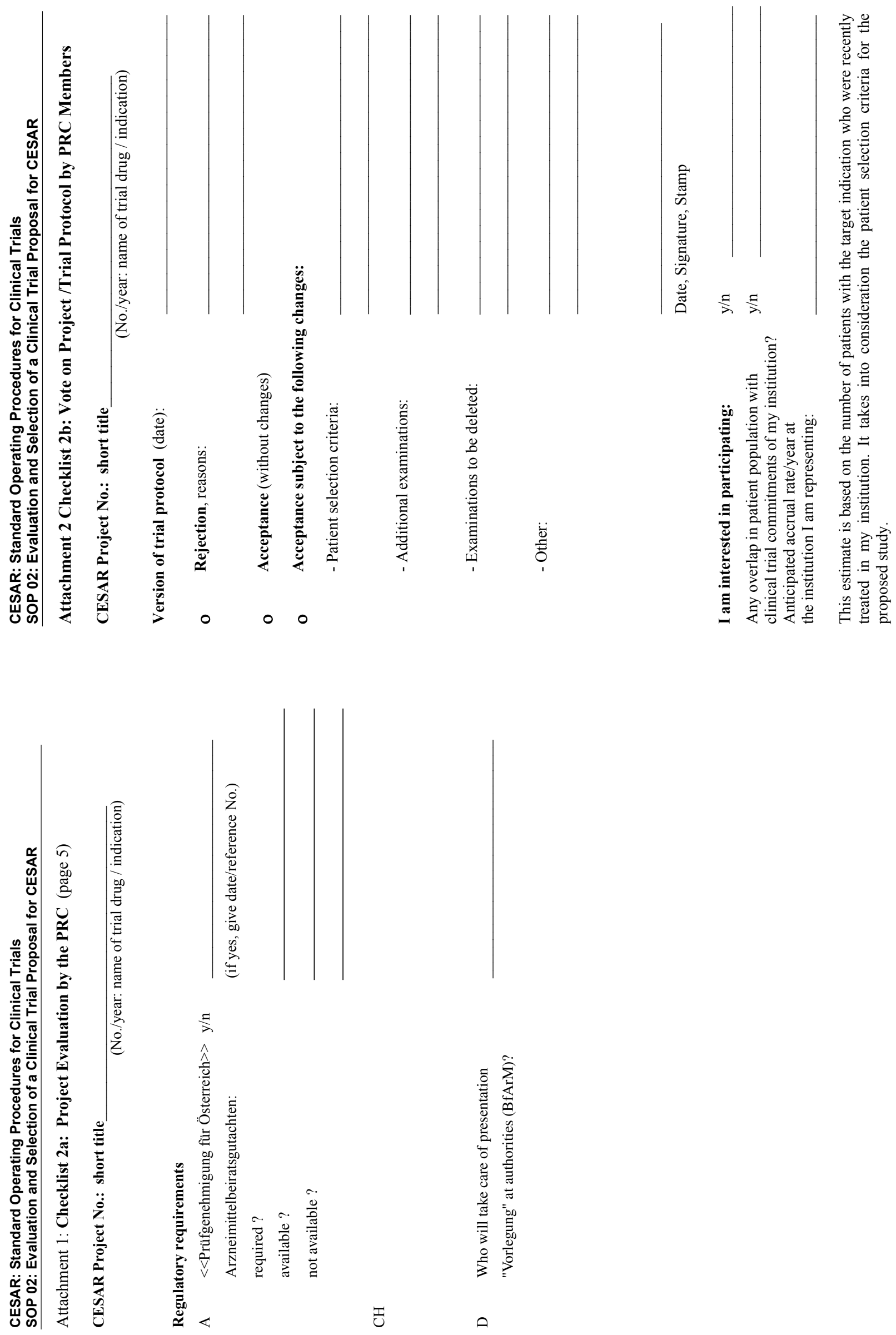
CESAR: Standard Operating Procedures for Clinical Trials

SOP 02: Evaluation and Selection of a Clinical Trial Proposal for CESAR

Attachment 3:

\section{Flow of information during protocol review}

The following graph illustrates the flow of information during the review process:

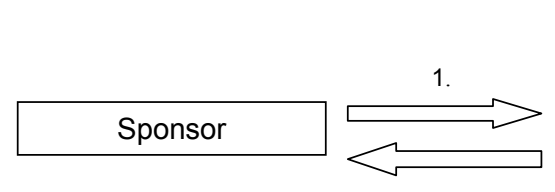

6.

3.

PRC members
Charimen of CESAR's Phase I-III Working Groups

Members of CESAR's Phase I-III Working Groups

2. $\sqrt{5}$

Chairman of the PRC

4. 